D. M. Glowacka ${ }^{1}$ - M. Crane $^{1}$ - D. J.

Goldie $^{1}$. S. Withington ${ }^{1}$

\title{
A Fabrication Route for Arrays of Ultra-Low-Noise MoAu Transition Edge Sensors on thin Silicon Nitride for Space Applications.
}

18.07.2011

Keywords Transition Edge Sensor, bolometer, far-infrared imaging array, space telescope

Abstract We describe a process route to fabricate arrays of Ultra-Low-Noise MoAu Transition Edge Sensors (TESs). The low thermal conductance required for space applications is achieved using $200 \mathrm{~nm}$-thick Silicon Nitride $\left(\mathrm{SiN}_{\mathrm{x}}\right)$ patterned to form long-thin legs with widths of $2.1 \mu \mathrm{m}$. Using bilayers formed on $\mathrm{SiN}_{\mathrm{x}}$ islands from films with $40 \mathrm{~nm}$-thick $\mathrm{Mo}$ and Au thicknesses in the range 30 to $280 \mathrm{~nm}$ deposited by dc-sputtering in ultra-high vacuum we can obtain tunable transition temperatures in the range 700 to $70 \mathrm{mK}$. The sensors use largearea absorbers fabricated from high resistivity, thin-film $\beta$-phase Ta to provide impedance-matching to incident radiation. The absorbers are patterned to reduce the heat capacity associated with the nitride support structure and include Au thermalising features to assist the heat flow into the TES. Arrays of 400 detectors at the pixel spacing required for the long-wavelength band of the far-infrared instrument SAFARI are now being fabricated. Device yields approaching 99\% are achieved.

PACS numbers: $85.250 \mathrm{Oj}, 95.55 \mathrm{Fw}$

\section{Introduction}

The far-infrared (FIR) band from $300 \mu \mathrm{m}$ to $20 \mu \mathrm{m}$ is of crucial importance for astrophysics, particularly with regard to studying the formation and evolution of galaxies, stars and planetary systems. It encompasses the wavelength range of the planned space missions SPICA ${ }^{\frac{1}{}}$, FIRI ${ }^{2}$ SAFIR, SPECS, and SPRIT; and it covers the wavelength range of the balloon interferometers BETTII $\underline{\underline{3}}$ and FITE. $\underline{4}$ The new generation of space missions covering this range will require large-format

1:Detector and Optical Physics Group, Cavendish Laboratory,

University of Cambridge, J. J. Thomson Avenue, Cambridge, CB3 OHE, UK

Tel.:+1223 337366

E-mail: d.m.glowacka@mrao.cam.ac.uk 
arrays of extremely low noise detectors with Noise Equivalent Powers (NEPs) of order $10^{-19} \mathrm{~W} / \sqrt{\mathrm{Hz}}$ or better. This requirement has driven the development of sensitive superconducting detectors, such as Transition Edge Sensors (TESs). While it is technically feasible to manufacture single TESs having this sensitivity, it is challenging to create an ultra-low-noise TES technology that can be engineered into complete imaging arrays, with the required optical packing and uniformity of performance. The NEPs required for these sensitive detectors can be

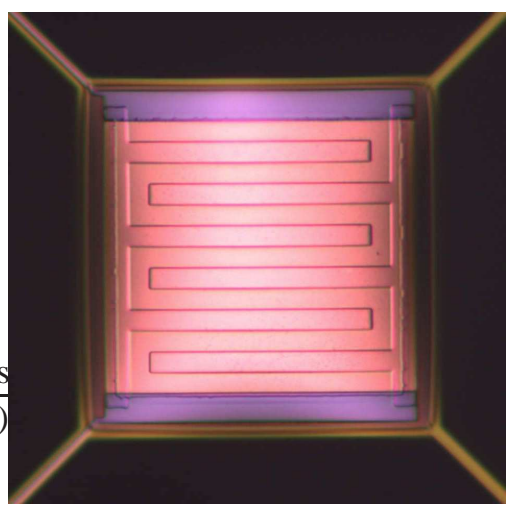

(a)

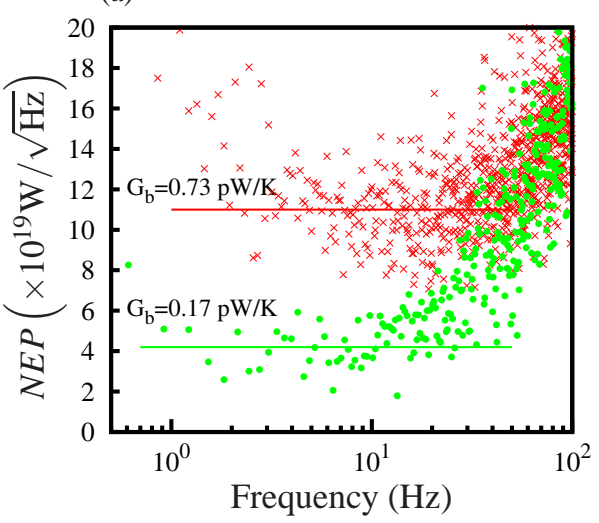

(b)

Fig. 1 (Color online) (a) Optical image of an earlier MoCu TES. (b) Dark NEP for an earlier $\mathrm{MoCu}$ TES reported in Ref. [7].

achieved by thermally isolating the TES using four long ( $\geq 500 \mu \mathrm{m})$ and narrow $(\leq 4 \mu \mathrm{m})$ support legs fabricated using thin $\operatorname{SiN}_{\mathrm{X}}$. We have previously made and characterized small arrays of MoCu TESs with $T_{c} \sim 100 \mathrm{mK}$ both with and without IR absorbers as part of a development effort to move from microstrip-coupled polarization-sensitive TESs with $N E P \sim 2 \times 10^{-17} \mathrm{~W} / \sqrt{\mathrm{Hz}}$ tailored for groundbased astronomy 5,6 to the ultra-low-noise performance required for space applications. ${ }^{7,8}$ Figure 1(a) shows an image of one of these $\mathrm{MoCu}$ devices fabricated 
without an additional absorber structure. The curvature in the central $\mathrm{SiN}_{\mathrm{x}}$ island is of order $1 \mu \mathrm{m}$ out of the figure and arises from residual stress in the $\mathrm{SiO}_{2}$ layer needed to passivate the $\mathrm{Cu}$. The $\mathrm{SiO}_{2}$ also contributes heat capacity and hence possibly additional noise features. MoAu TESs do not require passivation and our initial devices reported elsewhere in these proceedings 9 have demonstrated excellent long-term stability. Figure 1(b) shows the measured dark NEP for two of these TESs. The low frequency $N E P \sim 4 \times 10^{-19} \mathrm{~W} / \sqrt{\mathrm{Hz}}$ for a conductance to the bath $G_{b}=0.17 \mathrm{pW} / \mathrm{K}$ is within a factor of order $\sqrt{2}$ of the phonon limit calculated with noise modifier $\gamma_{\phi}=0.5 . \underline{10}$

In this paper we describe the fabrication route for ultra-low-noise MoAu TES detectors operating close to $100 \mathrm{mK}$ with a brief report on the status of the latest measurements.

\section{Experimental details}

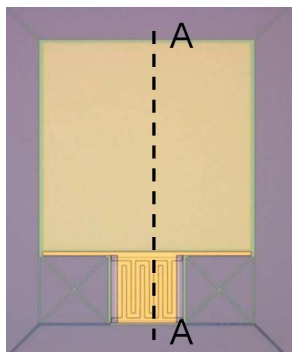

(a)

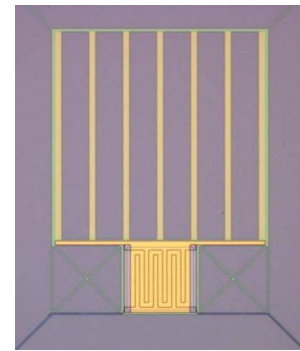

(b)

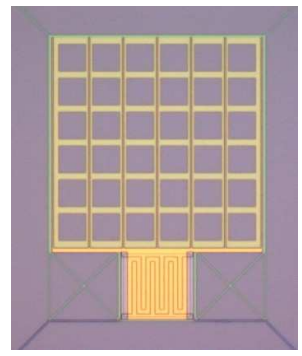

(c)

Fig. 2 (Color online) Optical images of:(a) A single $100 \times 100 \mu \mathrm{m}^{2} \mathrm{Mo} / \mathrm{Au}$ TES with longitudinal and partial lateral Au bars across the bilayer and Ta absorber. The $200 \mathrm{~nm}$ thick $\mathrm{SiN}_{x}$ island under the TES has an area $110 \times 110 \mu \mathrm{m}^{2}$. The Ta absorber is $320 \times 320 \mu \mathrm{m}^{2}$. The supporting legs are $2 \mu \mathrm{m}$ wide and $540 \mu \mathrm{m}$ long. (b) A striped absorber with seven $25 \mu \mathrm{m}$-wide Ta bars and (c) a meshed Ta absorber patterned into a $25 \mu \mathrm{m}$-wide grid to investigate the effect on the heat capacity.

The array described here has 388 single devices each consisting of a MoAu bilayer TES with Au banks and fingers partially across the TES, 11 a Ta FIR absorber, $\mathrm{Au}$ thermalizing structures and $\mathrm{Nb}$ connections all formed on a $\mathrm{SiN}_{\mathrm{x}}$ island. The island is thermally isolated from the $\mathrm{Si}$ wafer by four $\mathrm{SiN}_{\mathrm{x}}$ legs of width $2.1 \mu \mathrm{m}$ and length $540 \mu \mathrm{m}$, which determine the thermal conductance and hence the responsivity, saturation power and readout noise spectrum of the device. Photographs of the device types are shown in Fig. 2 .

The sensors are manufactured on $50 \mathrm{~mm}$ diameter, $225 \mu \mathrm{m}$ thick $\langle 100\rangle$, doubleside polished Si wafers. These wafers have, on both sides, a $50 \mathrm{~nm}$ film of thermal $\mathrm{SiO}_{2}$ and a $200 \mathrm{~nm}$ film spell of slightly off-stochiometric $\mathrm{SiN}_{\mathrm{x}}$, formed by lowpressure chemical vapour deposition. Ultimately, the $\mathrm{SiN}_{\mathrm{x}}$ becomes a suspended membrane, produced by back-side etching of the supporting silicon substrate under the TES and absorber, to give the required thermal isolation. 


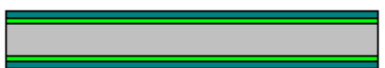

(a)

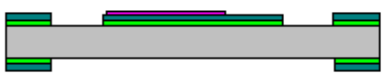

(c)

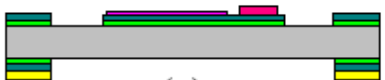

(e)

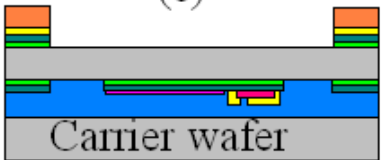

(g)

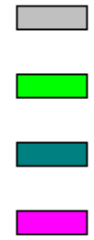

Si wafer

$\mathrm{SiO}_{2}$

$\mathrm{SiN}_{\mathrm{x}}$

Ta absorber

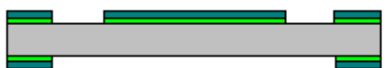

(b)

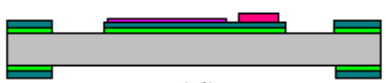

(d)

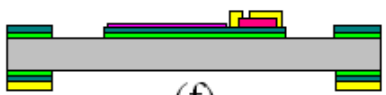

(f)

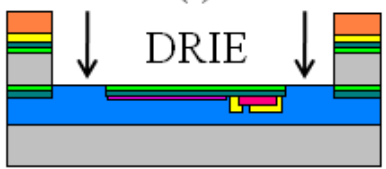

(h)

Fig. 3 (Color online) Schematic diagram showing the process flow for a single array. The cross section is along the line marked A-A in Fig. 2(a)

The first two steps of device fabrication are Reactive Ion Etching (RIE) of the $\mathrm{SiN}_{\mathrm{x}} / \mathrm{SiO}_{2}$ using $\mathrm{CHF}_{3}$ gas to outline the array and define the membrane and legs (done in two separate steps, one on the front of the wafer and one on the back): Fig. 3 a) and (b). Then the Ta absorber layer is deposited by DC magnetron sputtering in a system with a base pressure of $10^{-10}$ Torr as in Fig. 3 (c). The next step is deposition of superconducting bilayer by DC magnetron sputtering also under ultra-high vacuum conditions. The bilayer described here consists of a $40 \mathrm{~nm}$ Mo layer followed by a $195 \mathrm{~nm}$ Au layer, deposited in quick succession to avoid interface contamination and thereby achieving a reproducible proximity effect. The bilayer is patterned by wet etching: first the Au in a 50\% solution of commercially prepared gold etch and water, and then the Mo in a commercially prepared aluminum etch: Fig. 3 (d). The next processing step is the deposition of a $200 \mathrm{~nm}$-thick Au thermalizing layer on the back of the wafer: Fig. 3] e). A second $200 \mathrm{~nm}$ Au deposition forms thermalizing bars on the Ta absorber, and Au banks and lateral bars on the bilayer to improve thermalization of the TES: Fig. 3 f). A AuCu deposition, not shown, forms resistive heaters on the Si frame and Johnson noise thermometers on selected $\mathrm{SiN}_{\mathrm{x}}$ islands in place of the TES. The TES electrical connections and contact pads are formed from $250 \mathrm{~nm}$ of $\mathrm{Nb}$ (not shown on the process flow). At each stage, the Au and Nb layers are removed from unwanted areas by lift-off. The wafer is then ready for bonding face down to 
a carrier wafer: Fig. 3 $\mathrm{g}$ ). The last step is the fabrication of the membrane, which requires removal of the supporting Si from the window using Deep Reactive Ion Etching, thus leaving the TES and absorber membrane suspended on the nitride legs: Fig. 3(h). Finally the wafer is demounted, cleaned in an $\mathrm{O}_{2}$ plasma and is ready for testing.

The pixel spacing for the prototype array was matched to the SAFARI L-band requirement of $1.6 \mathrm{~mm}$. The array includes full Ta absorbers as in Fig. 2(a), striped (Fig. 2(b) and meshed absorbers (Fig.2(c)) . On selected calibration pixels the Ta was omitted completely and on others both the absorber and its support nitride was removed. Johnson noise thermometers were also included to assist with estimates of stray-light. The target $T_{c}$ was in the range $110-120 \mathrm{mK}$. The array was cooled in a closed-cycle, sorption-pumped dilution refrigerator mounted on a pulse-tube cooler giving a base temperature of $68 \mathrm{mK} \cdot \frac{12}{2}$ The array was enclosed in a Au-plated $\mathrm{Cu}$ box the inside of which was coated with light-absorbing $\mathrm{SiC}$ granules and carbon black mixed in Stycast 2850 to minimize scattered light. A photograph of the experimental enclosure is shown in Fig. 4(b) The sample space was surrounded by multiple layers of $\mathrm{Nb}$ foil and Metglas to provide magnetic shielding. We used NIST SQUIDs with analogue electronics readout that keeps the multiplexer in a fixed state but none-the-less permits readout of multiple channels. For these tests the SQUIDs and bias resistors have a separate light-tight enclosure with light-tight electrical feedthrough to the array enclosure.

\section{Results and Discussion}

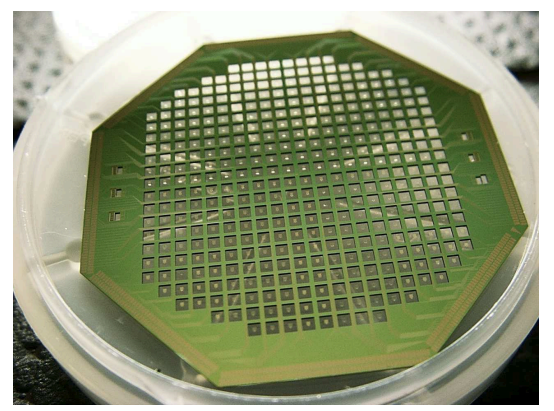

(a)

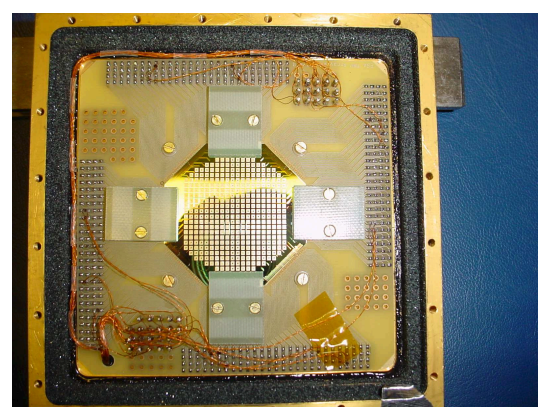

(b)

Fig. 4 (Color online) (a) Completed array chip. (b) Complete array mounted in a light-tight enclosure for low temperature testing.

The first 388-element arrays have now been completed and low temperature measurements are in progress. Thermal cycle tests already indicate that the mounting scheme shown in Fig.4(b) is robust and no devices have been damaged on repeated thermal cycles despite the removal of a large fraction of the support $\mathrm{Si}$ (of order 36\%). Overall device yield was 99\%. All TESs are clearly visible in Fig.4(a) including in the lower left portion a very small test pixel where the absorbing $\mathrm{Ta}$ 
and support $\mathrm{SiN}_{\mathrm{X}}$ was removed and the striped absorbers in the lower right sector. On a subset of TESs normal state resistances were measured to be $R_{n}=31 \pm 1 \mathrm{~m} \Omega$ and $T_{c}=113 \pm 5 \mathrm{mK}$. Conductance to the heat bath was $0.19 \pm 0.2 \mathrm{pW} / \mathrm{K}$ giving a predicted phonon-limited NEP of $2.4 \times 10^{-19} \mathrm{~W} / \sqrt{\mathrm{Hz}}$ calculated with noise modifier $\gamma_{\phi}=0.5$.

Further measurements are in progress to assess uniformity of the characteristics across the array, to measure in detail the dark NEP and response times as a function of absorber geometry, and to use the frame heaters to assess wafer heat sinking and pixel-to-pixel thermal cross-talk. We are also developing a fully micro-machined wafer backing-plate incorporating metallized back shorts and wiring fanout for integration into a full focal-plane. Results will be reported separately. The results already obtained suggest an important step-forward in the development of the next generation of space-based TESs and from the perspective of fabrication a significant step.

\section{Acknowledgments}

This work was supported in part by ESA Technology Research Programme Contract No. 22359/09/NL/CP. We are also very grateful to colleagues working on that contract within the Astronomy Instrumentation Group, Cardiff University, the Space Research Organization Netherlands, the National University of Ireland, Maynooth and the Space Science and Technology Department of Rutherford Appleton Laboratory for numerous stimulating discussions.

\section{References}

1. B. Swinyard, et al., Procs. SPIE, 2650, L2650, (2006).

2. F. P. Helmich and R. J. Ivison, Exp. Astron. 23, 245 (2009).

3. S. Rinehart and the BETTII Team, Procs. SPIE 7734, 77340K (2010).

4. T. Kanoh, et al., in Exoplanets and disks: their formation and diversity, AIP Conf. Procs. 1158, 389 (2009).

5. M. D. Audley, et al. in, 21st International Symposium on Space and Terahertz Technology, pp 76-84 (2010).

6. D. M. Glowacka, D. J. Goldie, S. Withington, M. Crane, V. Tsaneva, M. D. Audley and A. Bunting, J. Low Temp. Phys. 151, 249, (2008).

7. D. J. Goldie, A. Velichko, D. M. Glowacka and S. Withington, J. Appl. Phys. 109, 083105, (2011).

8. D. M. Glowacka, D. J. Goldie and S. Withington, in: 21st International Symposium on Space and Terahertz Technology, pp 276-279 (2010).

9. D. J. Goldie, A. Velichko, D. M. Glowacka and S. Withington, (These proceedings), (2012) DOI:10.1007/s10909-012-0575-x.

10. J. C. Mather, Appl. Optics 21, 1125 (1982).

11. J. N. Ullom, et al., Appl. Phys. Lett. 84, 4206 (2004).

12. G. Teleberg, S. T. Chase and L. Piccirillo, J. Low Temp. Phys. 151, 669, ((2008). 